\title{
Cutting, breaking, and tearing verbs in Hindi and Tamil ${ }^{*}$
}

BHUVANA NARASIMHAN

\section{Abstract}

Tamil and Hindi verbs of cutting, breaking, and tearing are shown to have a high degree of overlap in their extensions. However, there are also differences in the lexicalization patterns of these verbs in the two languages with regard to their category boundaries, and the number of verb types that are available to make finer-grained distinctions. Moreover, differences in the extensional ranges of corresponding verbs in the two languages can be motivated in terms of the properties of the instrument and the theme object.

Keywords: cut and break; separation events; verb semantics; Hindi; Tamil.

\section{Introduction}

In this article, I examine the encoding of the semantics of events of cutting, breaking, and tearing in two South Asian languages, Hindi (Indo-European, North India) and Tamil (Dravidian, South India). Although the two languages are genetically unrelated, they share linguistic traits partly owing to language contact over the centuries (Masica 2001), and a number of comparative studies have investigated specific aspects of the lexis and morphosyntactic structure of selected languages from the two language families (Ganesan 1976; Hook 2001; Lakshmi Bai 1986; Lust et al. 2000; among many others). The present study complements this literature with an investigation of the lexical categorization of a particular semantic domain in Hindi and Tamil. 


\section{Lexicalization patterns}

\subsection{Overall patterns}

In accordance with the overall crosslinguistic patterns of encoding events involving separation in the material integrity of objects (discussed in Majid et al., this issue), Hindi and Tamil are similar in distinguishing events based on the predictability of the location of the separation in the actedupon object. Cutting events involve a relatively high degree of predictability, whereas events of breaking involve greater uncertainty as to the locus of separation on the object. Hindi and Tamil speakers also distinguish between events of tearing (involving two-dimensional flexible objects) from events of both cutting and breaking in their descriptions of such events. Unlike speakers of many other languages, speakers of both Hindi and Tamil tend to group together events of snapping and smashing by labeling both types of events with the same verb.

These facts suggest that the Hindi and Tamil verbs corresponding to English cutting, breaking, and tearing might have equivalent semantics, and can be used interchangeably to describe similar situations. In this study, I show that along with the similarities there are also differences in the lexicalization patterns in the two languages, specifically, in the category boundaries of corresponding verbs in Hindi and Tamil, and in the availability of additional verbs to make finer-grained distinctions. Differences in the extensional ranges of corresponding verbs in the two languages can be motivated in terms of the properties of the instrument and the object acted upon (theme) by the agent.

The data for the present study were collected using an elicited production task (Bohnemeyer, Bowerman, and Brown 2001). Three Hindi and two Tamil speakers described videoclips of different types of cutting, breaking and tearing events (e.g., cutting a carrot with a knife; breaking a twig with the hands; tearing cloth). The particular verbs used to describe caused separation occurred either as the main verb or as a participial verb in the clause, e.g., the equivalents of He broke the twig or Breaking the twig, he put it down. Depending on the constructional and lexical resources of their language, speakers can vary their perspective on a given event, using an active, transitive construction to describe the event (e.g., She broke the twig) or an intransitive or passive construction to foreground the affected participant and the end result (e.g., the twig broke; the twig was broken). To maintain maximal comparability between the two languages, speaker variability in choice of event perspective was controlled for by selecting responses containing active, transitive constructions only. This led to the exclusion of seven responses coded as 
"other" (see Table 1). The "other" responses included five tokens of two intransitive verbs ( $k a T$ 'cut.intr.' 'become cut'; TuuT 'break.intr.' 'become broken'), one response containing a passive (the verb kaaT 'cut' used in a passive construction), and one missing response.

The resulting descriptions contained a total of 119 verb tokens in Hindi and 84 verb tokens in Tamil. ${ }^{1,2}$ The total number of verb types is eight in Hindi and nine in Tamil. ${ }^{3}$ The response types, when ordered according to frequency of use for both languages, show that verbs corresponding to English cut, break, and tear are among the most frequent verbs used by speakers of Hindi (kaaT 'cut'; toD| 'break'; phaaD| 'tear') and Tamil (veTTU 'cut'; oD $a i$ 'break'; kiZii 'tear'). ${ }^{4}$ Since the aim of the present study is to compare the extensional ranges of common verbs of cutting, breaking, and tearing in Hindi and Tamil, only those clips were selected which were described by at least one speaker from either language using one of the three high frequency causative verbs corresponding to cutting, breaking, or tearing.

A number of other, less frequent verb types were also used to label these clips. The Hindi speakers used (compound) verbs such as TukD|e kar 'cause to be in pieces', hisse kar 'cause to be in parts', phoD| 'shatter', thakuus/thakuuc 'smash', ciir 'split, tear $2^{\prime} .5$ The Tamil speakers used tuNDaa aakkU 'cause to be in pieces', narUkkU 'cut 2 ', arU 'saw, cut (thread/rope)', pirii 'open, separate', piyyii 'pull off/apart', kiir $U$ 'scratch, scrape', and kiiral pooTTU veyyii 'finish putting a scratch (in something)'.

Table 1 shows the distribution of verbs used to describe cutting, breaking, and tearing clips from the three speakers of Hindi (columns 2, 3, 4 in Table 1) and two speakers of Tamil (columns 5, 6 in Table 1). Extensional patterns of use of verbs corresponding to English cut, break, and tear (Hindi kaaT, toD $\mid$ and phaaD|; Tamil veTTU, oD $\mid a i$ and kiZii) cover large swathes of the domain. However, there are differences between the two languages in the category boundaries of these verbs and in the areas of semantic space where fine-grained differentiations are made with low frequency verb types.

\subsection{Breaking}

Both Hindi and Tamil speakers apply the break verb in their respective languages to overlapping scenes in videoclips involving the caused material separation of physical objects. However, the boundaries of the category vary in the two languages based on properties of the instrument and the object acted upon (the theme). The break verb in both languages is applied to clips involving rigid objects (e.g., pot, plate, branch) and blunt instruments (hand, hammer). The Hindi verb toD $\mid$ glossed here as 
Table 1. Verb distributions: cutting, tearing, and breaking in Hindi and Tamil.

Stimulus description (videoclip number)

Break stick over knee several times w/ intensity (5) Smash a stick into several fragments w/ 1 hammer blow (31)

Snap twig w/ 2 hands, but it doesn't come apart (25) Snap twig w/ 2 hands (19)

Snap carrot w/ 2 hands (57)

Smash plate w/ 1 hammer blow (40)

Smash carrot into several fragments w/ hammer (21)

Smash flower pot w/ 1 hammer blow (39)

Cut carrot in half crossways w/ 1 karate chop of hand (32)

Break vertically held stick w/ 1 karate chop of hand (42) Break yarn into many pieces w/ fury (35)

Break single piece off yarn by hand (38)

Chop rope stretched between 2 tables w/ a few hammer blows (50)

Break rope stretched between 2 tables w/ 1 karate chop of hand (61)

Cut carrot crossways into two pieces w/ 1 chisel blow (43)

Break stick in two w/ 1 downward chisel blow (53)

Cut rope stretched between 2 tables w/ 1 downward chisel blow (2)

Cut rope stretched between 2 tables w/ blow of axe (13) Chop branch repeatedly w/ axe until piece comes off (48) Cut carrot across into 2 pieces w/ 2 sawing motions of knife (26)

Cut rope in $2 \mathrm{w} / \mathrm{knife}$ (49)

Slice carrot across into multiple pieces w/ knife (10)

Slice carrot lengthwise w/ knife into 2 pieces (9)

Cut rope in $2 \mathrm{w} /$ scissors (24)

Cut cloth stretched between 2 tables in $2 \mathrm{w} /$ scissors (56)

Saw stick propped between two tables in half (15)

Cut single branch off twig w/ sawing motion of knife (20)

Cut hair w/ scissors (27)

Cut carrot in half crosswise w/ 1 blow of axe (54)

Cut fish into 3 pieces w/ sawing motion of knife (28)

Hack branch off tree w/ machete (3)

Make single incision in melon w/ knife (14)

Split melon in $2 \mathrm{w} /$ single knife blow (51)

Cut carrot in half lengthwise w/ 1 blow of axe (37)

Chop multiple carrots crossways w/ big knife, intensity (6)

Cut finger accidentally while cutting orange (18)

Cut strip of cloth stretched between 2 people's hands in 2 (12)

Chop cloth stretched between 2 tables w/ 1 karate chop of hand (34)

Chop cloth stretched between 2 tables w/ repeated knife blows (4)

Chop cloth stretched between 2 tables in 2 w/ 2 hammer blows (23)

Tear cloth into 2 pieces by hand (1)

Tear cloth about halfway through w/ 2 hands (36)

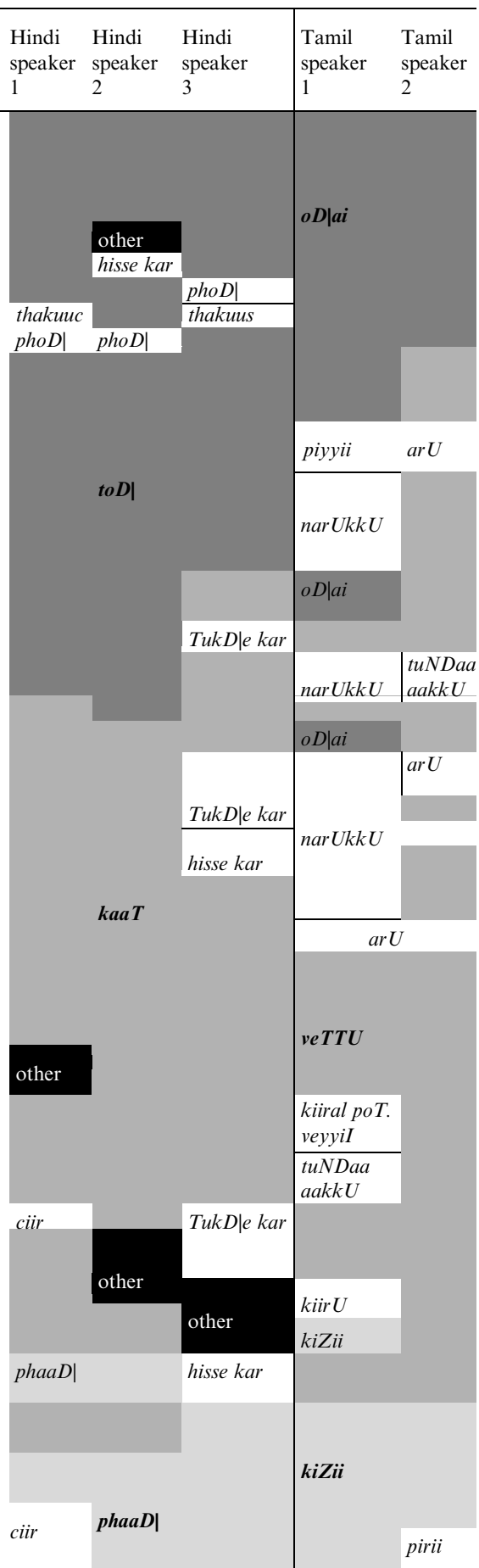


'break' is habitually used in situations described by the English verbs snap, break (something) up, demolish, fracture, split and typically involves rigid objects to which pressure is applied to create a breach in their integrity. The break might occur at the point at which the instrument makes contact with the object (e.g., the point at which a stroke of a hammer falls) or when contact is applied elsewhere on the object (e.g., the two ends of a stick or piece of yarn which snaps in the middle as a result of pressure applied from the ends).

Whereas Hindi speakers extend the use of $t o D \mid$ 'break' to non-rigid objects and instruments with blade-like characteristics as well, Tamil speakers' use of $o D \mid a i$ 'break' is more restrictive. The meaning of the Tamil verb $o D \mid a i$ is similar to that of Hindi $t o D \mid$. But unlike the Hindi speakers, both speakers of Tamil apply $o D \mid a i$ 'break' only to placement events involving rigid objects. They are also more conservative about the properties of the instrument relative to the Hindi speakers. One speaker restricts the use of $o D \mid a i$ solely to blunt instruments (e.g., hand, hammer). The second speaker extends uses of $o D \mid a i$ to clips involving instruments with a flat blade-like surface (e.g., hand spread out to execute a karatechop, chisel), but these constitute a subset of the clips labeled by Hindi speakers with $t o D \mid$.

In Hindi, some of the clips involving smashing (e.g., plate, pot), which are labelled by the $t o D \mid$ verb, are also described using the verb phoD| which describes a specific type of breaking event: one involving a certain degree of violence and resulting in the theme object being shattered into several pieces. Where the object is rigid (e.g., carrot), but not as brittle as a plate or a pot, the (dialectal) verb thakuuc(s) 'smash' is employed by two of the Hindi speakers. Tamil speakers do not differentiate these smashing clips from the other types of breaking clips, labeling them all with the verb $o D \mid a i$.

\subsection{Cutting}

The Hindi verb kaaT 'cut' is typically used for events described in English with verbs such as cut, carve, slash, slice, hack, bite, sting, and implies use of a (bladed or pointed) instrument. Clips involving (single or double) sharp-bladed instruments such as a knife, scissors or axe are described using the verb kaaT by at least two out of the three Hindi speakers, irrespective of whether the theme object is extended along one dimension (wool, rope) or two dimensions (cloth), and whether it is flexible (hair, rope) or rigid (branch, carrot). One speaker also uses kaaT for clips involving use of a chisel point. 
Unlike the Hindi verb kaaT, the Tamil verb veTTU cannot be used to mean 'bite' or 'sting' even though these imply sharp or pointed instruments (e.g., teeth); a different verb kaDii is used for such actions. But veTTU, like toD|, can also be used for theme objects varying in dimensionality and flexibility. Further, as discussed in the previous section, the verb veTTU has a wider range of application than Hindi kaaT, and extends not only to clips involving a sharp-bladed instrument, but also to clips with instruments construable as having an edge (e.g., edge of the hand, chisel) which, in Hindi, are labeled with $t o D \mid$. Tamil speakers also differ from speakers of Hindi in splitting the domain of cutting more finely, using specific verbs such as $\operatorname{ar} U$ 'saw or cut thread/rope', narUkkU 'cut ${ }_{2}$ ', and kiirU 'scratch, scrape'.

The motivation for some of the finer-grained distinctions made by Tamil speakers in the verbs used to describe the cutting clips is relatively clear. The verb arU can be applied to events that involve a particular manner of cutting with a bladed instrument, viz. cutting with a knife using sawing motions. It can also be applied to events that involve neither a particular manner of cutting (sawing) nor a bladed instrument, but have to do with creating a separation in one-dimensional flexible artifacts (e.g., wool, thread, but not hair). In this sense, the verb ar $U$ has something in common with the verb piyyii 'pull apart/off' which is used for actions of pulling apart flexible (one-, two-, or three-dimensional) objects. The actions described by piyyii also involve a certain degree of force that is required to cause a separation in the theme object. It cannot be described for scenes such as take top off a pen or take lid off can which are described using torai 'open' by Tamil speakers. Both piyyii and arU are similar to $o D \mid a i$ 'break' in not requiring use of a bladed instrument; but they also have something in common with the cutting and tearing verbs veTTU and kiZii, both of which can be used with non-rigid theme objects.

For events that involve incomplete cutting (making a cut in a finger or melon), the verb kiirU 'scrape, scratch' is used, which can be used to focus on the use of a pointed or sharp-edged instrument to make a separation in an entity with pre-existing divisions (e.g., coconut pulp from the shell), or to create a separation in an entity which is whole (e.g., a melon, a fingertip). It is harder to pin down the precise semantic specifications of the verb narukkU. At best, it could be analyzed as meaning 'cut nonbrittle (small) object (into bits) with a bladed instrument'. This specification is needed to account for its extension to objects such as a carrot, cloth, and rope which differ in rigidity but share the property of not being brittle and the ability to bend (unlike, for instance, a plate). Presumably cutting hair with scissors does not qualify for use of narUkkU (or $\operatorname{ar} U$ ) 
because the use of veTTU for cutting hair is strongly conventionalized. However, a second possibility is that the clip involving cutting hair focused on cutting off hair and retaining the uncut hair, whereas narUkkU cares about the end state of the theme (the cut off pieces). The use of nar$U k k U$ for clips involving non-bladed instruments (e.g., hammer, edge of hand) suggests that use of a bladed instrument is not crucial for use of this verb; however there are too few uses (and only by one speaker) to arrive at a satisfactory characterization of this verb.

Hindi speakers also use expressions other than kaaT for the cutting domain, but these are typically semantically more general than the verb $k a a T$, including TukD|e kar 'cause to be in pieces' and hisse kar 'cause to be in parts' which specify the resulting state of the theme objects and not the manner, type of theme object, or the instrument involved in the action. Speakers of Tamil also use semantically general complex predicates of this type (tuNDaa aakU 'cause to be in pieces'), but they do so less frequently. Hence, the lexicalization of the cutting domain in Hindi is both "super-general" relative to Tamil, as well as more circumscribed in its boundaries with the neighbouring region of break.

\subsection{Tearing}

Both Hindi and Tamil distinguish tearing verbs from cutting and breaking verbs. The most frequent tear verb (Hindi phaaD $\mid$ and Tamil kiZii) is applied to a relatively small proportion of the clips; all of which involve causing a material separation in a two-dimensional flexible object (e.g., cloth). There is, however, some variability at the boundary. Hindi speakers prefer to use the verb kaaT even when a two-dimensional flexible theme object is involved when the instrument used is sharp-bladed (e.g., scissors, knife), switching to use of phaaD| for all other types of instruments. The motivation for Tamil speakers' alternations between kiZii versus veTTU/narUkkU is less clear. While kiZii is applied to events involving cloth as the acted-upon object, not all cloth clips are labeled with this verb. Where the cloth is cut in several strokes with scissors, or involves punctual separation using an instrument with an edge (a single knife stroke or a karate stroke with the edge of the hand), Tamil speakers use the verb veTTU. However, if the action of cutting the cloth involves several degrees of separation using a knife or the hands, for example, owing to the number of cutting strokes required, or the prolonged interval of tearing with the hands, the verb kiZii is used. Other verbs in this domain include ciir 'split, tear, in Hindi (used to describe tearing cloth using hands) and pirii 'separate' in Tamil (used to describe tearing cloth partways with the hands). Interestingly, the Tamil speakers also extend the 
verb pirii to opening events where the theme object is not split into parts but is only partially separated (e.g., open a book, open scissors).

\section{Discussion}

The lexicalization patterns in Hindi and Tamil are characterized by a high degree of overlap in their patterns of extension suggesting shared similarity in meaning. But we also find variation between speakers of the same language. ${ }^{6}$ For instance, Hindi speaker 3 appears to differ considerably from speakers 1 and 2, largely by virtue of using compound verbs more often. It might be argued that this variation arises because speakers have different understandings of verbs such as kaaT 'cut' and toD| 'break'. However, the intensional meaning of the compound verbs and the locii of variation suggest that speakers are adopting different construals on the same scene. Compound expressions such as TukD|e kar 'cause to be in pieces' and hisse kar 'cause to be in parts' specify the end result of the theme object but not the nature of the causal action. The speaker can thus felicitously extend such verbs to scenes where a cutting or breaking verb may not prototypically apply or to scenes that are amenable to more than one event construal. Interestingly, compound verb uses by Hindi speaker 3 occur at category boundaries between breaking and cutting verbs (break stick in two with one downward chisel blow), and between cutting and tearing verbs (chop cloth stretched between two tables with one karate chop of hand). They also occur in descriptions of clips where there is inter-speaker variability in verb use in Tamil as well, suggesting that the relevant clips can be construed in more than one way. For instance, Tamil speakers' uses of arU 'saw, cut (thread/rope)' and narUkkU 'cut ${ }_{2}$ ' (for the scene cut carrot across into two pieces with two sawing motions of knife) suggest a focus on the manner of cutting (sawing) versus the end state of the theme (being in cut-off pieces) and its properties (bendable, lack of brittleness). Alternations between uses of veTTU and narUkkU (for scenes such as cut rope in two with knife and slice carrot lengthwise with knife into two pieces) might also reflect differences in the degree of focus on the properties of the theme (veTTU is applied to a wider range of themes than narUkkU). Similarly, uses of piyyii 'pull apart' and $\operatorname{ar} U$ 'saw, cut (thread/rope)' for scenes involving the separation of yarn show that speakers can describe either the manner of causing a separation (involving resistance) or choose to specify the the properties of the theme (one-dimensional flexible object) that is being acted upon in the placement event. These differences highlight the importance of understanding the intensions of the verbs that are used in the clip descriptions, 
since overlap in verb extension does not necessarily reflect overlap in intension.

The present study shows that the extensional patterns of corresponding verbs in Hindi and Tamil are broadly similar, yet reveal differences at a more fine-grained level: in the category boundaries of frequent verbs, in the stock of other verbs available for describing such events, and in the regions of semantic space where finer semantic distinctions are made. The Hindi and Tamil data suggest that patterns of variability in the category boundaries of corresponding high frequency verbs in the two languages are not random, but can be motivated in terms of the properties of the theme object and the type of instrument, and in some cases, the manner in which the action is carried out.

Received 8 December 2004

Revision received 3 August 2006
Max Planck Institute for Psycholinguistics, The Netherlands

\section{Notes}

* Postal address: Max Planck Institute for Psycholinguistics, Postbus 310, 6500 AH Nijmegen, The Netherlands. Author's e-mail address〈bhuvana@mpi.nl >.

1. Transliteration conventions for Spoken Tamil (ST) (based partly on Schiffman 1999): Short vowels are represented by $a, i, u, e$, o, and long vowels by $a a, i i, u u, e e, o o$ and $a e$ (typically used in English borrowings). Orthographic $u$ and $i$ are often pronounced with an unrounded centralized high vowel, represented here as $U$. In ST, this vowel is also added word-finally when the word does not end in a vowel. Diphthongs include ai (pronounced as a monophthong in some contexts in ST and represented by $E$ ) and $a u$. A short vowel such as $a$ followed by a word-final nasal in ST is pronounced [õ] and represented by $O$. Consonants include $p, t, T$ (retroflex), $c$, and $k$ (and the voiced counterparts: $b, d, D$ (retroflex), $j, g$ ), $m, n, N$ (retroflex), $r, Z$ (voiced, retroflex approximant), $l, L$ (retroflex), $s, s h, h, y$, and $v$. Geminate consonants are represented by doubling and flapped consonants by a "" symbol following the consonant.

2. Transliteration conventions for Hindi (based partly on McGregor 1995): Short vowels are represented by $a, i, u, e, o$ and long vowels by $a a, i i, u u, e e$, and $o o$. The diphthongs are represented by $a u$ and $a e$. Nasal vowels are represented using capital letters corresponding to the letters used to represent oral vowels. Consonants include $k$, $k h$ (aspirated), $g, g h$ (aspirated), $c, c h$ (aspirated), $j, j h$ (aspirated), $t, t h$ (aspirated), $d, d h$ (aspirated), $T$ (retroflex), $T h$ (aspirated, retroflex), $D$ (retroflex), $D h$ (aspirated, retroflex), $p$, $p h$ (aspirated), $b, b h$ (aspirated), $m, n, N$ (retroflex), $y, r, l, w, s, s h, h$. Geminate consonants are represented by doubling and flapped consonants by a "l" symbol following the consonant.

3. In two cases, the Hindi verbs were treated as pronunciation variants of the same verb: toD $\mid$ and thuuD $\mid$ and thakuuc and thakuus. My secondary Hindi informant could not identify the verbs thakuuc and thakuus. It is possible that these are dialectal forms since they were provided by two speakers of Hindi from the same area of Samastipur, Bihar; 
whereas the third speaker was from Delhi. These verbs were given a preliminary gloss: 'smash' based on the stimulus scene to which they were applied (smashing a carrot with a hammer).

4. The glosses for the Tamil and Hindi verbs are based on dictionary definitions as well as consultations with two Tamil native speakers (from Thanjavur and Pudukottai, Tamil Nadu, India) and one Hindi native speaker (New Delhi, India).

5. The full utterance containing complex expressions such as TukD|e kar 'cause to be in pieces' and hisse kar 'cause to be in parts' often include quantificational specifications (e.g., do hisse kar 'cause to be in two pieces' or kaii TukD|e kar 'cause to be in several pieces'); these additional modifications are ignored for current purposes.

6. I am grateful to two anonymous reviewers for comments on this issue.

\section{References}

Bohnemeyer, Jürgen, Melissa Bowerman, and Penelope Brown

2001 Cut and break clips. In Levinson, Stephen C., and Nick J. Enfield (eds.), Field Manual 2001, Language and Cognition Group, Max Planck Institute for Psycholinguistics. Nijmegen: MPI, 90-96.

Ganesan, S. N.

1976 Case constructions in Tamil and Hindi: A contrastive study. In Agesthialingom, Shunmugom, and K. Kushalappa Gowda (eds.), Dravidian Case System. Annamalainagar: Annamalai University, 367-404.

Hook, Peter E.

2001 Where do compound verbs come from? (And where are they going?). In Bhaskararao, Peri, and Karumuri Venkata Subbarao (eds.), Yearbook of South Asian Languages and Linguistics: Tokyo Symposium on South Asian Languages (Contact, Convergence, and Typology). New Delhi/Thousand Oaks/London: Sage Publications, 101-130.

Lakshmi Bai, B.

1986 A note on syntactic convergence among Indian languages: The verb 'to be'. In Krishnamurti, Bhadriraju (ed.), South Asian Languages: Structure, Convergence, and Diglossia. Delhi: Motilal Banarsidass, 195-208.

Lust, Barbara, Kashi Wali, James Gair, and Karumuri Venkata Subbarao

2000 Introduction to lexical anaphors and pronouns in selected South Asian languages. In Lust, Barbara, Kashi Wali, James Gair, and Karumuri Venkata Subbarao (eds.), Lexical Anaphors and Pronouns in Selected South Asian Languages. Berlin/New York: Mouton de Gruyter, 1-46.

Masica, Colin

2001 The definition and significance of linguistic areas: Methods, pitfalls, and possibilities (with special reference to the validity of South Asia as a linguistic area). In Bhaskararao, Peri, and Karumuri Venkata Subbarao (eds.), Yearbook of South Asian Languages and Linguistics: Tokyo Symposium on South Asian Languages (Contact, Convergence and, Typology). New Delhi/ Thousand Oaks/London: Sage Publications, 205-267.

Majid, Asifa, Melissa Bowerman, Miriam van Staden, and James S. Boster

this issue The semantic categories of cutting and breaking events: A crosslinguistic perspective. Cognitive Linguistics 18(2), 133-152. 
McGregor, R. S.

1995 Outline of Hindi Grammar. Oxford, UK: Oxford University Press.

Schiffman, Harold F.

1999 A Reference Grammar of Spoken Tamil. Cambridge, UK: Cambridge University Press. 
